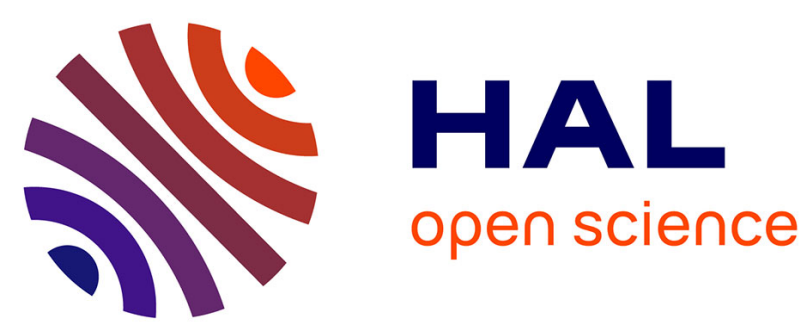

\title{
Theoretical and numerical study on high frequency vibrational convection: Influence of the vibration direction on the flow structure
}

Samia Bouarab, Faiza Mokhtari, Slim Kaddeche, Daniel Henry, Valéry Botton, Abdessamed Medelfef

\section{To cite this version:}

Samia Bouarab, Faiza Mokhtari, Slim Kaddeche, Daniel Henry, Valéry Botton, et al.. Theoretical and numerical study on high frequency vibrational convection: Influence of the vibration direction on the flow structure. Physics of Fluids, 2019, 31 (4), pp.043605. 10.1063/1.5090264 . hal-02356325

\section{HAL Id: hal-02356325 \\ https://hal.science/hal-02356325}

Submitted on 8 Nov 2019

HAL is a multi-disciplinary open access archive for the deposit and dissemination of scientific research documents, whether they are published or not. The documents may come from teaching and research institutions in France or abroad, or from public or private research centers.
L'archive ouverte pluridisciplinaire HAL, est destinée au dépôt et à la diffusion de documents scientifiques de niveau recherche, publiés ou non, émanant des établissements d'enseignement et de recherche français ou étrangers, des laboratoires publics ou privés. 


\title{
Theoretical and numerical study on high frequency vibrational convection: influence of the vibration direction on the flow structure
}

\author{
Samia Bouarab ${ }^{1,2}$, Faiza Mokhtari ${ }^{1}$, Slim Kaddeche $^{3}$, Daniel Henry ${ }^{4}$, Valéry Botton ${ }^{4,5}$, \\ Abdessamed Medelfef ${ }^{1,4}$ \\ ${ }^{1}$ Laboratoire de Thermodynamique et Systèmes Energétiques, Faculté de Physique, Université des Sciences et de \\ la Technologie Houari Boumediène, BP 32, 16111 Bab Ezzouar, Alger, Algeria \\ ${ }^{2}$ Université M'hammed Bougara, Boumerdes, Algeria \\ ${ }^{3}$ Laboratoire de Recherche Matériaux, Mesures et Applications, Institut National des Sciences Appliquées et de \\ Technologie, BP 676, 1080 Tunis Cedex, Tunisia \\ ${ }^{4}$ Laboratoire de Mécanique des Fluides et d'Acoustique, CNRS, Université de Lyon, \\ Ecole Centrale de Lyon/ Université Lyon 1/INSA de Lyon, \\ ECL, 36 Avenue Guy de Collongue, 69134 Ecully Cedex, France \\ ${ }^{5}$ INSA Euro-Méditerranée, Université Euro-Méditerranéenne de Fès, Route de Meknès, BP51, Fez, Morocco
}

\begin{abstract}
Thermal convection induced simultaneously by horizontal temperature gradient and vibration in a rectangular cavity filled with molten silicon is investigated numerically and theoretically. The time averaged equations of convection are solved in the high-frequency vibration approximation. The Chebyshev spectral collocation method and a Newton-type method based on the Frechet derivative are used in the numerical solution of the streamfunction formulation of the incompressible Navier-Stokes equations. Validation by comparison with previous works has been performed. Different values of the Grashof number Gr and vibrational Grashof number $\mathrm{Gr}_{\mathrm{v}}$ and all the possible orientations of the vibrations are considered. Numerical results show that depending on the vibration direction, the flow can be amplified or damped, with even the possibility of flow inversion which can occur between critical vibration angles $\alpha_{1}$ and $\alpha_{2}$. A general theoretical expression is derived relating these critical angles and the ratio of vibrational to buoyant convection parameters, $\mathrm{Gr}_{\mathrm{v}} / \mathrm{Gr}$. A very good agreement between the theoretical and numerical results is obtained.
\end{abstract}

\section{Introduction}

When a fluid with a temperature gradient is subjected to external vibration, a supplementary flow, known as thermovibrational convection, can be generated and this flow will transport heat and matter as does buoyant convection. Vibrations can then be used to suppress or strengthen buoyant convection depending on the vibration orientation and the thermal or compositional gradients [1]. Buoyancy and vibration induced flows are characterized by two dimensionless parameters, the Grashof $(\mathrm{Gr})$ and vibrational Grashof $\left(\mathrm{Gr}_{\mathrm{v}}\right)$ numbers. $\mathrm{Gr}_{\mathrm{v}}$ depends on the amplitude of the vibration, the angular frequency and the applied temperature difference. The relative importance of thermovibrational and buoyant convection mechanisms is defined by the ratio $\mathrm{Gr}_{\mathrm{v}} / \mathrm{Gr}$. Gershuni and Lyubimov [2] summarized the theory of thermovibrational convection with high and finite frequency vibrations in closed and infinite cavities. For small amplitude and very high-frequency vibration, the period of vibrations is small in comparison with the reference viscous and heat/mass diffusion times, which permits us to use the averaging technique and obtain the set of equations governing the averaged heat and flow fields. Gershuni and Lyubimov [2] showed that when the direction of vibration is perpendicular to the temperature gradient under weightlessness, a non-zero mean flow exists at any value of the vibration amplitude. 
There have been extensive studies of thermovibrational convection in weightlessness and ground conditions in various configurations [1-19]. These studies are often motivated by the difficulties faced during crystal growth, solidification of molten alloys and other related industrial processes. These difficulties concern the control of the composition of the final solid material, particularly the level of impurities, which is connected with the control of the natural convection and the heat and mass transfer in the melt during the solidification process. And the use of vibrations is an interesting non-intrusive means to achieve this control.

The main research works on thermovibrational convection have used the model developed by Gershuni and Lyubimov [2], but have applied it in situations of increasing complexity, from 1D model configurations to fully 3D complex situations. Gresho and Sani [3] and Gershuni et al. [4] were the first to investigate the effect of sinusoidal gravity modulation in a differentially heated fluid layer through linear stability analysis. They found that the gravity modulation can significantly influence the stability of a system by increasing or decreasing its susceptibility to convection and that stabilization can be obtained for small amplitude vertical oscillations.

Gershuni et al. [5] presented the first numerical results of thermovibrational convection in rectangular and square cavities in weightlessness. Numerical results of gravity modulation effect on thermal convection in microgravity and terrestrial conditions have been reported by Biringen and Peltier [6]. They made a more general nonlinear three-dimensional analysis and confirmed the pioneering results of Gresho and Sani [3] and Gershuni et al. [4]. Natural heat convection suppression in semiconductor melts with the help of horizontal vibration is studied by Uspenskii and Favier [7] in vertical and horizontal Bridgman configurations. They compared the efficiency of fluid motion damping under magnetic field and vibrations and found that the damping is more efficient with the magnetic field for the horizontal Bridgman geometry.

Thermovibrational convection in a cubic fluid cell has been investigated by Savino, Monti and Piccirillo [8]. A qualitative similarity between their three-dimensional results and the previous two-dimensional simulations has been found. Lan [9] found that the axial vibration can be effective for the control of melt flows and growth interface in vertical zone-melting of GaAs.

A theoretical study has been conducted by Straube et al. [10] on the average dynamics of various two-phase systems subjected to high-frequency transversal vibration using the continuum approach. They developed a theoretical model for a suspension of nondeformable particles for the pulsation and averaged dynamics, analyzed the stability of a quasi-equilibrium state in a plane layer and studied vibrational dynamics of compressible bubbly fluids. The effect of vibration parallel to an imposed temperature gradient in a two-phase stratified liquid system is analyzed by Chang et al. [11] using a hybrid thermal lattice Boltzmann method. The ability of the applied vibration to enhance the flow, the heat transfer and the interface distortion is investigated. They compare the response of a two-phase stratified fluid system with a singlephase fluid system.

O'Hern et al. [12] performed experiments in a thin quasi-two-dimensional rectangular cell filled with silicone oil subjected to vertical vibrations. They showed that the upper gas-liquid interface breaks up into jets and droplets under strong vertical vibrations. Bubbles are also generated, which move downward against buoyancy towards the bottom of the container and eventually form a large gas cavity which is stably levitated below another gas-liquid interface.

Experimental and computational studies of thermal vibrational convection in the reducedgravity environment of a parabolic flight have been performed by Shevtsova's research team [13-15]. Some of the experiments [13] were carried out in a cubic cell with differentially heated walls, which was filled with isopropanol and subjected to translational vibration in the direction perpendicular to the temperature gradient. Two numerical approaches were employed: two- 
dimensional simulations on the basis of averaged equations describing the mean fields and three-dimensional modelling on the basis of full Navier-Stokes and heat transfer equations. The authors found a very good agreement between experimental results and three-dimensional numerical simulations with real microgravity profile recorded by the on-board accelerometer.

The dynamical behavior of an isothermal liquid bridge under a step change in the mass force magnitude has been studied by Ferrera et al. [14]. Their numerical results show the same shape and amplitude of the free surface oscillations as those found experimentally and they point out that the liquid bridge is more sensitive to lateral than to axial perturbations.

More recently, Gaponenko et al. [15] performed an experimental and numerical study of the stability of two superimposed miscible liquids with different densities under horizontal vibrations. They observe the development of interfacial columnar patterns, the characteristics of which depend on the width of the diffusive transient zone at the interface. They also find a qualitative agreement between the numerical results and the parabolic flight experiments.

Particle accumulation structures under the effect of g-jitters were studied by Lappa [16] with the express intent of supporting the optimization of future experiments to be performed in space. He showed that the resulting degree of turbulence depends essentially on the direction, amplitude and frequency of the applied inertial disturbance. More recently, Lappa [17] investigated the interaction of different types of flow, induced by buoyancy, Marangoni effect and vibrations, as a possible means to control convection structure and intensity in shallow rectangular cavities filled with a low Prandtl number liquid. Lappa showed that the effect of a vertical magnetic field is qualitatively similar to that induced by high-frequency vibrations with direction parallel to the temperature gradient. In a second paper, Lappa [18] analyzed the impact of hybrid convection induced by the joint influence of imposed vibrations and surface-tensioninduced forces in a nonisothermal liquid layer. He identified the regimes of quasi-stationary rolls, standing waves, traveling waves, and modulated disturbances. Traveling waves can reverse their direction of propagation in some specific regions of the phase space.

In the current work, we want to assess the possibility to control natural convection and consequently heat and mass transfer by using vibrations, in view of using it during directional solidification processes. We have in mind crystal growth applications, but also the purification of metallurgical grade silicon in order to get silicon suitable for photovoltaic applications. For that, we present a theoretical and numerical study on the influence of high frequency vibrations on convection induced in a crucible with an aspect ratio $A=$ length/height $=4$ containing a melt at low Prandtl number $(\mathrm{Pr}=0.01)$ subjected to a horizontal thermal gradient. Different values of the Grashof number Gr and the vibrational Grashof number $\mathrm{Gr}_{\mathrm{v}}$ for all possible directions of the vibrations are investigated. The focus of the work is to describe and analyze the changes of the flow patterns with the vibration direction. In the next section, the studied configuration is described and the governing equations for high-frequency vibration are derived with the appropriate boundary conditions. The numerical method and tests of validation are then presented. The theoretical and numerical results are finally discussed before some concluding remarks.

\section{Mathematical formulation}

In this section, we describe the mathematical model used for the numerical simulation of buoyant convection in a melt submitted to high frequency vibrations.

The geometry of the problem is shown in figure 1. It consists of a rectangular crucible of height $H$ and length $L$ filled with molten silicon, a low-Prandtl-number fluid with $\operatorname{Pr}=0.01$. The 
aspect ratio for this cavity is defined as $A=L / H$. The vertical sidewalls are thermally conducting and maintained at fixed temperatures, $T_{h}$ at the right hot wall and $T_{c}$ at the left cold wall, while the horizontal top and bottom walls are adiabatic. The flow which develops in the fluid due to the horizontal thermal gradient resulting from the differentially heated sidewalls is assumed to be laminar.

A vibration corresponding to a sinusoidal displacement $\boldsymbol{s}(t)=b \sin (\Omega t) \boldsymbol{n}$ is applied to the crucible containing the melt. It induces an acceleration $\boldsymbol{g}(t)=b \Omega^{2} \sin (\Omega t) \boldsymbol{n}$. In these expressions, $b$ is the amplitude of the vibration, $\Omega=2 \pi f$ is the angular frequency where $\mathrm{f}$ is the frequency, and $\boldsymbol{n}$ is the unit vector indicating the direction of the vibration, which makes an angle $\alpha$ with the horizontal in the $(x, z)$ plane. This angle $\alpha$ will be often called vibration angle in the following.

The Navier-Stokes equations governing the flow in the melt have then to include the effect of both the static gravity acceleration $\boldsymbol{g}_{\mathbf{0}}$ and the additional vibrational component. The resulting acceleration $\boldsymbol{g}$ is then:

$$
\boldsymbol{g}=\boldsymbol{g}_{0}+b \Omega^{2} \sin (\Omega t) \boldsymbol{n}
$$

For a Newtonian, incompressible fluid and in the Boussinesq approximation (the density variation may be taken into account only in the buoyant force, with a linear temperature dependence), the set of governing equations takes the form

$$
\begin{gathered}
\frac{\partial \boldsymbol{V}}{\partial t}+(\boldsymbol{V} . \boldsymbol{\nabla}) \boldsymbol{V}=-\frac{1}{\rho_{0}} \boldsymbol{\nabla} p+v \boldsymbol{\nabla}^{2} \boldsymbol{V}+\beta\left(T-T_{0}\right) g \boldsymbol{\gamma}+\beta\left(T-T_{0}\right) b \Omega^{2} \sin (\Omega t) \boldsymbol{n}, \\
\frac{\partial T}{\partial t}+\boldsymbol{V} \cdot \boldsymbol{\nabla} T=\chi \nabla^{2} T \\
\nabla . \boldsymbol{V}=0,
\end{gathered}
$$

where $\boldsymbol{V}, T, p$ and $t$ are velocity, temperature, pressure and time, respectively. $T_{0}$ is a reference temperature, $T_{0}=\left(T_{h}+T_{c}\right) / 2$, and $\rho_{0}$ is the density at $T_{0} . \beta, v$ and $\chi$ are the thermal expansion coefficient, the kinematic viscosity and the thermal diffusivity, respectively. $\gamma$ is the unit vector in the vertical $z$ direction.

In the limiting case of high-frequency harmonic vibrations, Gershuni and Lyubimov [2] propose to use an averaging method for studying what they call thermovibrational convection. For that, the period $\tau$ of the vibrations must be much smaller than both the reference viscous and thermal diffusion times $\left(\tau \ll \min \left(H^{2} / \nu, H^{2} / \chi\right)\right)$ and the displacement of fluid particles during one period of oscillation must also be much smaller than the characteristic length scale taken here as the crucible height $H$. Each convective flow characteristic, velocity, temperature and pressure, can be decomposed into a high frequency oscillatory part (with a characteristic time $\tau=2 \pi / \Omega$ ) and a slow evolution part (steady or low frequency oscillatory) corresponding to the average on the vibration period $\tau$, which evolves with a characteristic time much larger than $\tau$.

In the averaging method, the decomposed variables are introduced in the equations (2-4). These equations limited to the fast terms are first solved analytically to get expressions of the fast variables (pulsation velocity and temperature) as a function of slow variables. Then by averaging the complete set of equations on a vibration period and using the expressions of the 
fast variables, the system of equations governing the slow evolution flow can be obtained. Note that an assessment of the thermovibrational theory with a general and complete derivation of the asymptotic equations for various regimes can be found in Savino and Lappa [19]. Using $H$, $H^{2} / v, v / H, \Delta T / A=\left(T_{h}-T_{c}\right) / A$ and $\rho v^{2} / H^{2}$ as scales for length, time, velocity, temperature and pressure (the temperature is now $\left(T-T_{0}\right) A / \Delta T$ ), and keeping the same notations for the average flow characteristics, these equations in dimensionless form are

$$
\begin{gathered}
\frac{\partial \boldsymbol{V}}{\partial t}+(\boldsymbol{V} . \boldsymbol{\nabla}) \boldsymbol{V}=-\boldsymbol{\nabla} p+\nabla^{2} \boldsymbol{V}+\operatorname{Gr} T \boldsymbol{\gamma}+\operatorname{Gr}_{\mathrm{v}}(\boldsymbol{W} \cdot \boldsymbol{\nabla})(T \boldsymbol{n}-\boldsymbol{W}), \\
\frac{\partial T}{\partial t}+\boldsymbol{V} . \boldsymbol{\nabla T}=\frac{1}{\operatorname{Pr}} \nabla^{2} T, \\
\nabla . \boldsymbol{V}=0, \\
\nabla . \boldsymbol{W}=0, \quad \nabla \times \boldsymbol{W}=\boldsymbol{\nabla T} \times \boldsymbol{n}
\end{gathered}
$$

$\boldsymbol{W}$ is the solenoidal part of the vector field $T \boldsymbol{n}$. It verifies Eqs. (8) and is made dimensionless in the same way as the temperature. As indicated by Gershuni and Lyubimov [2], it is also the proper amplitude of the pulsation velocity. The parameters involved in these equations are the Prandtl number Pr, the Grashof number Gr and the vibrational Grashof number $\mathrm{Gr}_{\mathrm{v}}$, defined respectively as

$$
\operatorname{Pr}=\frac{v}{\chi}, \mathrm{Gr}=\frac{g \beta \Delta T H^{4}}{L v^{2}} \text { and } \mathrm{Gr}_{\mathrm{v}}=\frac{(b \Omega \beta \Delta T)^{2} H^{4}}{2 L^{2} v^{2}}
$$

No-slip boundary conditions are applied on all the crucible walls, giving $\boldsymbol{V}=0$. Concerning $\boldsymbol{W}$, due to the neglect of the viscous force when deriving the pulsation velocity, a nonpermeability condition may be imposed rather than the no-slip condition, i.e. $\boldsymbol{W}_{n}=0$ where $\boldsymbol{W}_{n}$ is the component of $\boldsymbol{W}$ normal to the wall. The temperature is imposed on the left and right sidewalls and the top and bottom walls are adiabatic. In a dimensionless form, this gives $T=$ $-A / 2$ at $x=-A / 2, T=A / 2$ at $x=A / 2$, and $d T / d z=0$ at $z=-1 / 2$ and $z=1 / 2$.

For this two-dimensional study, it is more convenient to use a stream function formulation. Two stream functions $\psi$ and $F$ associated with the velocity $\boldsymbol{V}$ and the pulsation velocity component $\boldsymbol{W}$ can be defined:

$$
V_{x}=-\frac{\partial \psi}{\partial z}, \quad V_{z}=\frac{\partial \psi}{\partial x}, \quad W_{x}=-\frac{\partial F}{\partial z}, \quad W_{z}=\frac{\partial F}{\partial x} .
$$

Using the fact that the vibrational term in (5) can be written as $\operatorname{Gr}_{\mathrm{v}}(\boldsymbol{W} . \boldsymbol{n}) \boldsymbol{\nabla T}$ with some part included in the pressure (Gershuni and Lyubimov [2]) and taking the curl of (5), the complete set of equations we have to solve is given by

$$
\begin{aligned}
\frac{\partial \nabla^{2} \psi}{\partial t}+\left(\frac{\partial \psi}{\partial x}\right. & \left.\frac{\partial \nabla^{2} \psi}{\partial z}-\frac{\partial \psi}{\partial z} \frac{\partial \nabla^{2} \psi}{\partial x}\right) \\
& =\nabla^{2} \nabla^{2} \psi+\mathrm{Gr} \frac{\partial T}{\partial x} \\
& +\operatorname{Gr}_{\mathrm{v}}\left[\left(\frac{\partial^{2} F}{\partial x^{2}} \frac{\partial T}{\partial z}-\frac{\partial^{2} F}{\partial x \partial z} \frac{\partial T}{\partial x}\right) \sin \alpha-\left(\frac{\partial^{2} F}{\partial x \partial z} \frac{\partial T}{\partial z}-\frac{\partial^{2} F}{\partial z^{2}} \frac{\partial T}{\partial x}\right) \cos \alpha\right]
\end{aligned}
$$




$$
\begin{gathered}
\frac{\partial T}{\partial t}+\left(\frac{\partial \psi}{\partial x} \frac{\partial T}{\partial z}-\frac{\partial \psi}{\partial z} \frac{\partial T}{\partial x}\right)=\frac{1}{\operatorname{Pr}} \nabla^{2} T \\
\nabla^{2} F=\frac{\partial T}{\partial x} \sin \alpha-\frac{\partial T}{\partial z} \cos \alpha
\end{gathered}
$$

The boundary conditions for $\psi$ and $F$ associated with the equations (9-11) can be written as $\psi=0, \partial \psi / \partial x=0$ and $F=0$ at $x=-A / 2$ and $x=A / 2$, and $\psi=0, \partial \psi / \partial z=0$ and $F=0$ at $z=-1 / 2$ and $z=1 / 2$. Note that $\psi=0$ and $F=0$ correspond to the non-penetration condition for $\boldsymbol{V}$ and $\boldsymbol{W}$, respectively.

The vibration angle $\alpha$ is involved in the equations (9)-(11) through the quantities $\sin \alpha$ and $\cos \alpha$, i.e. with a $2 \pi$ period. In fact, if $\alpha$ is changed to $\alpha+\pi, \sin \alpha$ and $\cos \alpha$ are changed to their opposite, but, according to (11), $F$ will also be changed to its opposite. As a result, the vibrational part of the driving force, quantified by $\mathrm{Gr}_{\mathrm{v}}$ in (9), will not change, indicating an effective period of $\pi$. In the following, we will then choose to take $\alpha$ in the interval $[0, \pi]$ i.e. $\left[0^{\circ}, 180^{\circ}\right]$. We will also verify that the flows obtained at $\alpha=0^{\circ}$ and $180^{\circ}$, together with their variations around these values, are identical.

Note that the only symmetry existing in our two-dimensional problem is the central symmetry $S_{c}$, which is defined by

$$
S_{c}:(x, z) \rightarrow(-x,-z), \quad\left(V_{x}, V_{z}, T, F\right) \rightarrow\left(-V_{x},-V_{z},-T, F\right) .
$$

\section{Numerical method and validation}

In the current study, we take advantage of the highly accurate spectral collocation method [20] on Gauss-Lobatto-Chebyshev points to solve the set of equations (9-11) with the relevant boundary conditions. As explained by Trefethen [21], if $N$ is the number of discretization points, it is always possible to achieve a convergence rate of $O\left(N^{-m}\right)$ for every natural integer $m$ when using a spectral method, provided the solution is infinitely differentiable. Such a behavior is known as spectral accuracy. In contrast, for finite difference and finite element methods, the value of $m$ will depend on the order of approximation and the smoothness of the solution. Since our two-dimensional physical space is defined on the $[0, A] \times[0,1]$ domain, the unknown variables will be computed at shifted Gauss-Lobatto-Chebyshev points adapted to this domain. The variables are interpolated between these points through a series of Lagrange polynomials and the approximate values of the partial derivatives of these variables will be obtained thanks to differentiation matrices, as those proposed by Trefethen [21] and Weideman and Reddy [22].

In order to get a good rate of convergence, the steady state solution of the nonlinear system (911 ) is computed with a Newton-type method based on the Frechet derivative of the nonlinear operators, as proposed by Phillips [23].

Tests of validation and accuracy of our simulations have been done by comparisons of our results with those of Lappa [17]. He considered a cavity with an aspect ratio $A=4$ containing molten silicon $(\operatorname{Pr}=0.01)$. Using our definition of the parameters, the calculations were performed for $\mathrm{Gr}=2.510^{4}$, without vibration and with vibrations in horizontal and vertical 
directions $\left(\mathrm{Gr}_{\mathrm{v}}=3.12510^{5}\right)$. The comparisons are shown in Table 1 . For a grid $61 \times 51$, we see that there is a very good agreement between our results and those of Lappa [17] .

Table 1 Comparison of our results with those of Lappa [17] for $A=4, \mathrm{Gr}=2.510^{4}, \mathrm{Pr}=$ 0.01 , in cases without vibration $\left(\mathrm{Gr}_{\mathrm{v}}=0\right)$ and with vibrations $\left(\mathrm{Gr}_{\mathrm{v}}=3.12510^{5}\right)$ in horizontal and vertical directions.

\begin{tabular}{|c|c|c|c|}
\hline & $\mathrm{Gr}_{\mathrm{v}}=0$ & $\begin{array}{c}\mathrm{Gr}_{\mathrm{v}}=3.12510^{5} \\
\alpha=0^{\circ}\end{array}$ & $\begin{array}{c}\mathrm{Gr}_{\mathrm{v}}=3.12510^{5} \\
\alpha=90^{\circ}\end{array}$ \\
\hline Lappa (2016) & $\psi_{\max }=0.678$ & $\psi_{\max }=0.62$ & $\psi_{\max }=0.134$ \\
\hline Our results & $\psi_{\max }=0.678$ & $\psi_{\max }=0.616$ & $\psi_{\max }=0.131$ \\
\hline
\end{tabular}

\section{Results}

To study the effect of vibrations on convection, we have performed numerical simulations for a cavity with an aspect ratio $A=4$ and a fixed Prandtl number $\operatorname{Pr}=0.01$ corresponding to molten silicon. We have considered different values of the Grashof number $\mathrm{Gr}$ and vibrational Grashof number $\mathrm{Gr}_{\mathrm{v}}$ and all the possible orientations of the vibrations, for $\alpha$ between $0^{\circ}$ and $180^{\circ}$.

\subsection{Influence of the vibration direction}

A first series of numerical simulations has been performed for a fixed value of the Grashof number, $\mathrm{Gr}=2500$, two values of the vibrational Grashof number, $\mathrm{Gr}_{\mathrm{v}}=6.2510^{3}$ and $6.2510^{4}$, and regularly spaced values of the vibration angle $\alpha$ between $0^{\circ}$ and $180^{\circ}$. The results are first shown in figure 2 where, for the two values of $\mathrm{Gr}_{\mathrm{v}}$, the value $\psi_{c}$ of the stream function at the center of the cavity $(x=2, z=0.5)$ is plotted as a function of $\alpha . \psi_{c}$ will give an indication on the flow intensity in the central convective roll. Without vibrations, this central roll, which goes up at the right hot wall and down at the left cold wall, turns in the counterclockwise direction and occupies the whole cavity. It is associated with a negative value of $\psi_{c}$, more precisely $\psi_{c}=-6.29$, and the maximum value of the velocity horizontal component is $V_{x, \max }=19.75$. When vibrations are applied (figure 2), we see that $\psi_{c}$ strongly varies with the direction of the vibrations and can even change sign. More precisely, for $0^{\circ} \leq$ $\alpha \leq 90^{\circ}, \psi_{c}$ remains negative indicating a roll turning in the same counterclockwise direction as the pure thermal roll and this roll has its maximal intensity reached for $\alpha$ close to $45^{\circ}$. For $90^{\circ} \leq \alpha \leq 180^{\circ}$, in contrast, there are two values of $\alpha, \alpha_{1}$ and $\alpha_{2}$, for which $\psi_{c}$ vanishes, indicating a range $\left[\alpha_{1}, \alpha_{2}\right]$ where $\psi_{c}$ becomes positive. It means that, in this range of $\alpha$, there is a flow inversion and the central roll now turns in the clockwise direction. As shown in figure 2, when the vibrational Grashof number $\mathrm{Gr}_{\mathrm{v}}$ is increased, the maximum intensity of the counterclockwise roll is increased, as well as the size of the range $\left[\alpha_{1}, \alpha_{2}\right]$ where a clockwise flow appears and the maximum intensity of this clockwise roll.

Some more information on the effect of the vibration orientation in the two same cases is given in figures 3 and 4 with the plot of the maximal absolute horizontal velocity, together with insets showing the stream function inside the cavity for selected values of $\alpha$. These plots confirm that the global range of orientations from $\alpha=0^{\circ}$ to $\alpha=180^{\circ}$ can be divided in different regions. Note first that when vibration and imposed temperature gradient are in the same direction, i.e. 
for $\alpha=0^{\circ}$, the flow is less intense than the buoyant flow, indicating that the vibrations induce a damping effect in this case. In a first region $\left(0^{\circ} \leq \alpha \leq \alpha_{m}\right)$, the vibrations induce an intensification of the flow, corresponding to the decrease of the damping for small values of $\alpha$ and then to an acceleration effect, with a maximal intensification found for $\alpha=\alpha_{m}$. In a second region ( $\alpha_{m} \leq \alpha \leq \alpha_{1}$ ), we observe the decrease of the flow intensity, corresponding first to the decrease of the acceleration effect and then to a damping effect, leading to a complex weak flow for $\alpha=\alpha_{1}$. In a third region $\left(\alpha_{1} \leq \alpha \leq \alpha_{2}\right)$, we observe the reverse flow, corresponding successively to an increase and a decrease of a reverse acceleration effect and still leading to weak flows for $\alpha=\alpha_{2}$. Finally, for $\alpha_{2} \leq \alpha \leq 180^{\circ}$, we have the decrease of the damping effect, in continuity with what was found for $\alpha$ above $0^{\circ}$. Note that the plots of $\left|V_{x, \max }\right|$ do not give clear values for $\alpha_{1}$ and $\alpha_{2}$, as $\left|V_{x, \max }\right|$ can be associated with different rolls in the cavity.

In the first case, for $\mathrm{Gr}_{\mathrm{v}}=6.2510^{3}$ (figure 3), the structure of the flow is almost preserved in a large range of $\alpha$ values, with a single roll in the counterclockwise direction occupying the whole cavity. The maximum flow intensity is obtained for $\alpha \approx 42^{\circ}$. Structure changes only occur around the range $\left[\alpha_{1}, \alpha_{2}\right]$ of flow inversion. These changes can be observed in figure 3 (insets) and in figure 5 where velocity fields are plotted. At $\alpha=117^{\circ}$, with the decrease of the vibrational force in the central part of the cavity, the counterclockwise flow has split into two counterclockwise rolls in the end parts of the cavity. A reverse flow is further initiated in the central part, but, even at its maximum intensity $\left(\alpha \approx 135^{\circ}\right)$, this reverse flow will not occupy the whole cavity, counterclockwise rolls remaining in the upper right and lower left corners. The way back to the counterclockwise roll occurs in a similar way. At $\alpha=153.5^{\circ}$, the central part is stagnant and counterclockwise rolls are present in the end parts of the cavity. These two rolls will further merge for larger values of $\alpha$.

In the second case, for a larger vibrational intensity $\left(\mathrm{Gr}_{\mathrm{v}}=6.2510^{4}\right.$, figures 4 and 6$)$, more changes affect the structure of the flow. The intensification of the flow by vibrational effect up to $\alpha_{m} \approx 54^{\circ}$ leads to a global counterclockwise circulation around a concentrated counterclockwise roll, as obtained in pure buoyant flows with enough large Gr values. The decrease of the flow intensity for $\alpha$ up to $\alpha_{1}$ occurs through different structure changes. When the vibration direction is perpendicular to the temperature gradient, i.e. for $\alpha=90^{\circ}$, the global counterclockwise circulation has changed with the presence of two small clockwise vortices in the upper left and lower right corners and a counterclockwise diagonal vortex. Very small changes from this vertical vibration direction will then lead to main changes in the flow pattern. For $\alpha=92.3^{\circ}$, the counterclockwise circulation is now reduced to two vortices in the upper right and lower left corners, giving a flow structure with a stagnant core and four vortices in the end parts. And for $\alpha=93^{\circ}$, the two clockwise vortices have merged in a global clockwise circulation with still the two counterclockwise vortices. Note that this flow structure is almost the up-down symmetric of the flow structure at $\alpha=90^{\circ}$. For larger $\alpha$, the clockwise flow will develop and intensify. At its maximum intensity (close to $\alpha=128^{\circ}$ ), it has a structure similar to the counterclockwise flow at $\alpha_{m}$, but with a reverse rotation direction. This still gives an approximate up-down symmetry between these two structures. The next flow inversion near $\alpha_{2}$ will occur differently. The clockwise flow will decrease in intensity $\left(\alpha=176.4^{\circ}\right)$, then decrease in size with counterclockwise rolls appearing in the end parts $\left(\alpha=177.3^{\circ}\right)$, and finally disappear giving a stagnant core while the counterclockwise rolls develop in size and intensity $\left(\alpha=177.7^{\circ}\right.$ ). At $\alpha=180^{\circ}$, the two counterclockwise rolls have eventually merged giving a 
global counterclockwise circulation in the cavity. Note that the transition near $\alpha_{2}$ is somewhat similar to what was found for the smaller value of $\mathrm{Gr}_{\mathrm{v}}$.

The changes of the flow pattern when the vibration direction is modified are expected to be connected with the changes affecting the global driving forces due to gravity and vibration. In particular, the flow inversion observed around $\alpha=135^{\circ}$ must correspond to such forces in a direction opposite to those due to the sole gravity, allowing the hot fluid to go down and the cold fluid to go up. The same kind of effect may be involved in the experiment of O'Hern et al. [12] on silicon oil mentioned in the introduction, which depicts the descending movement of bubbles and the levitation of a gas cavity below a liquid zone when the container is subjected to strong vibrations. We will study that in more details in the following sections.

\subsection{Theoretical model accounting for flow inversion}

In the previous section, it has been shown that the vibrations can strongly change the convective flow induced by side-heating. Depending on the vibrations direction, the flow can be amplified or damped, with even the possibility of flow inversion. From the two previously considered cases $\left(\mathrm{Gr}=2500\right.$ and $\mathrm{Gr}_{\mathrm{v}}=6.2510^{3}$ and $\left.6.2510^{4}\right)$, the flow inversion is found to occur between critical vibration angles $\alpha_{1}$ and $\alpha_{2}$, which seem to depend on the characteristic parameters of the cases considered.

Our objective is to derive a general theoretical expression for these critical angles. For that, different observations can be made. First, these critical angles exactly correspond to flow inversion in the central part of the cavity, i.e. to situations with very weak flows in these zones, for which the global driving force must be almost zero. Using Eq. (9), we can then write

$$
\mathrm{Gr} \frac{\partial T}{\partial x}+\mathrm{Gr}_{\mathrm{v}}\left[\left(\frac{\partial^{2} F}{\partial x^{2}} \frac{\partial T}{\partial z}-\frac{\partial^{2} F}{\partial x \partial z} \frac{\partial T}{\partial x}\right) \sin \alpha-\left(\frac{\partial^{2} F}{\partial x \partial z} \frac{\partial T}{\partial z}-\frac{\partial^{2} F}{\partial z^{2}} \frac{\partial T}{\partial x}\right) \cos \alpha\right]=0
$$

These very weak flows are generally associated with a diffusive regime for the temperature. This is confirmed in figure $7 \mathrm{a}$, where the temperature field is plotted for the case $\mathrm{Gr}=2500$, $\mathrm{Gr}_{\mathrm{v}}=6.2510^{3}$ and a vibration angle $\alpha=117^{\circ}$, a value close to the critical angle $\alpha_{1}$ of flow inversion. We can then assume that

$$
\frac{\partial T}{\partial z}=0 \text { and } \frac{\partial T}{\partial x} \cong \frac{\Delta T}{\Delta x}=\frac{(A / 2)-(-A / 2)}{A}=1
$$

Some information are also needed for the stream function $F$ associated with the pulsation velocity component $\boldsymbol{W}$. For the same case close to the critical angle $\alpha_{1}$, the field of $F$ is plotted in figure $7 \mathrm{~b}$. We see that, in the central part of the cavity, $F$ is quite independent of $x$ and only depends on $z$. We can then assume that

$$
F=F(z) \text { and } \partial F / \partial x, \partial(\partial F / \partial x) / \partial x \text { and } \partial(\partial F / \partial z) / \partial x \text { are weak. }
$$

Moreover, using the assumptions given in Eqs. (13) and (14), Eq. (11) will give

$$
\frac{\partial^{2} F}{\partial z^{2}}=\sin \alpha
$$


Using all these particular properties of $T$ and $F$, only the term $\frac{\partial^{2} F}{\partial z^{2}} \frac{\partial T}{\partial x} \cos \alpha$ will remain in the vibrational part of the driving force and Eq. (12) will then be simplified to

$$
\mathrm{Gr}+\mathrm{Gr}_{\mathrm{v}}[\sin \alpha \cos \alpha]=0
$$

Note that Gershuni and Lyubimov [2] found a similar expression for the no-flow equilibrium condition in a plane fluid layer inclined at an angle $\alpha$ to the vertical and subjected to a horizontal temperature gradient and vertical vibrations.

A simple equation for the critical values of $\alpha$ can then be obtained, which is:

$$
\sin 2 \alpha_{c}=-2 \mathrm{Gr} / \mathrm{Gr}_{\mathrm{v}} \text {. }
$$

The expression of $\alpha_{c}(17)$ indicates that the critical angles only exist for $2 \frac{\mathrm{Gr}}{\mathrm{Gr}_{\mathrm{v}}} \leq 1$. This is then a condition for the observation of flow inversion, which can be written as

$$
\frac{\mathrm{Gr}_{\mathrm{v}}}{\mathrm{Gr}} \geq 2
$$

The equation (17) for $\alpha_{c}$ can be further resolved. In the case where the condition (18) is verified, we can define the angle $\theta_{0}$ such that

$$
\sin \theta_{0}=2 \mathrm{Gr} / \mathrm{Gr}_{\mathrm{v}}
$$

The general solutions for $\alpha_{c}$ are then given by

$$
2 \alpha_{c}=\pi+\theta_{0}+2 k \pi \text { and } 2 \alpha_{c}=-\theta_{0}+2 k \pi,
$$

which, in the range $\left[0^{\circ}, 180^{\circ}\right]$ considered here, gives

$$
\alpha_{1}=90^{\circ}+\theta_{0} / 2 \text { and } \alpha_{2}=-\theta_{0} / 2+180^{\circ} \text { with } \theta_{0}=\operatorname{asin}\left[2 /\left(\mathrm{Gr}_{\mathrm{v}} / \mathrm{Gr}\right)\right]
$$

Eq. (21) gives simple expressions for the vibration limit angles $\alpha_{1}$ and $\alpha_{2}$ defining the domain of flow inversion. In particular, we see that $\alpha_{1}$ and $\alpha_{2}$ are always between $90^{\circ}$ and $180^{\circ}$, that they are equally spaced from $90^{\circ}$ and $180^{\circ}$, respectively, and that the domain of flow inversion they determine increases when $\theta_{0}$ is small, i.e. when the ratio $\mathrm{Gr}_{\mathrm{v}} / \mathrm{Gr}$ is strong.

\subsection{Discussion on vibration effect and flow inversion}

In order to check the validity and the precision of the theoretical expressions for the critical vibration angles given in (21), we can first calculate the theoretical values for the two cases discussed in the previous section. In the first case $\left(\mathrm{Gr}=2500, \mathrm{Gr}_{\mathrm{v}}=6250, \mathrm{Gr}_{\mathrm{v}} / \mathrm{Gr}=2.5\right)$, we obtain $\alpha_{1}=116.565^{\circ}$ and $\alpha_{2}=153.435^{\circ}$ and in the second case $\left(\mathrm{Gr}=2500, \mathrm{Gr}_{\mathrm{v}}=\right.$ 
$6.2510^{4}, \mathrm{Gr}_{\mathrm{v}} / \mathrm{Gr}=25$ ), we obtain $\alpha_{1}=92.294^{\circ}$ and $\alpha_{2}=177.706^{\circ}$. These values are close to those obtained from the simulations, which are estimated from the condition $\psi_{c}=0$, first roughly using figure 2 and then more precisely with extra-calculations. Indeed, we obtain $\alpha_{1}=$ $116.8^{\circ}$ and $\alpha_{2}=153.3^{\circ}$ in the first case and $\alpha_{1}=92.3^{\circ}$ and $\alpha_{2}=177.7^{\circ}$ in the second case.

The expressions given in (21) also indicate that the range of vibration angles giving a flow inversion only depends on the ratio $\mathrm{Gr}_{\mathrm{v}} / \mathrm{Gr}$. To check that, series of computations have been performed for different Grashof and vibrational Grashof numbers giving the same ratio $\mathrm{Gr}_{\mathrm{v}} / \mathrm{Gr}$. Two ratios have been chosen, $\mathrm{Gr}_{\mathrm{v}} / \mathrm{Gr}=2.5$ and 25, both being larger than 2 in order to observe a flow inversion. The variation of the stream function at the center, $\psi_{c}$, with the vibration angle $\alpha$ is plotted in figures 8 and 9 for the two different ratios $\mathrm{Gr}_{\mathrm{v}} / \mathrm{Gr}$. At fixed ratio, we observe a general increase of the intensity of the flow in the main central roll when $\mathrm{Gr}_{\mathrm{v}}$ and $\mathrm{Gr}$ are increased. This increase of the flow is effective for all vibration angles, i.e. in both situations with the usual counterclockwise roll and with flow inversion, and then also affects the maxima of both counterclockwise and clockwise flows. Moreover, for each ratio, the curves of $\psi_{c}$ obtained in the different cases go to zero for the same values of $\alpha$, determining the same $\alpha$ range where flow inversion occurs. This range is still very well defined by the expressions of $\alpha_{1}$ and $\alpha_{2}$ given in (21) and the values obtained are those already indicated at the beginning of the section. Figures 8 and 9 also clearly show that the $\alpha$ range for flow inversion increases when the ratio $\mathrm{Gr}_{\mathrm{v}} / \mathrm{Gr}$ is increased, as it was expected from the expressions in (21).

Note that the maximum $\alpha$ range for flow inversion corresponds to $\left[90^{\circ}, 180^{\circ}\right]$ and is obtained for $\theta_{0}=0$, i.e. for very large values of the ratio $\mathrm{Gr}_{\mathrm{v}} / \mathrm{Gr}$. In that limit, we have a counterclockwise flow for $\alpha \in\left[0^{\circ}, 90^{\circ}\right]$ and a clockwise flow for $\alpha \in\left[90^{\circ}, 180^{\circ}\right]$. Moreover, as shown in figure 10 with cases corresponding to large values of $\mathrm{Gr}_{\mathrm{v}} / \mathrm{Gr}$, the curves of $\psi_{c}$ versus $\alpha$ eventually give clockwise flows with the same intensity as counterclockwise flows. In fact, it can be shown that, in the limit of large $\mathrm{Gr}_{\mathrm{v}} / \mathrm{Gr}$, the flow obtained for $\alpha=90^{\circ}+\theta$ is exactly the reverse flow of that obtained for $\alpha=90^{\circ}-\theta$ and corresponds to its symmetric with respect to the up-down symmetry (such approximate symmetry was observed in the previous section for $\mathrm{Gr}_{\mathrm{v}} / \mathrm{Gr}=25$ ). Indeed, in that limit, the vibrational part of the driving velocity is dominant and the buoyancy part can be neglected. We have then to show that, if $V_{x, 1}, V_{z, 1}, T_{1}$ and $F_{1}$ are the fields defined at $x_{1}$ and $z_{1}$ and solutions for $\alpha=90^{\circ}-\theta$ with the vibrational force $F_{v 1}$, the fields obtained by the up-down symmetry, $V_{x, 2}=V_{x, 1}, V_{z, 2}=-V_{z, 1}, T_{2}=T_{1}$ and $F_{2}=F_{1}$ at $x_{2}=x_{1}$ and $z_{2}=-z_{1}$ are solutions for $\alpha=90^{\circ}+\theta$ with the vibrational force $F_{v 2}=-F_{v 1}$. First, $F_{1}$ is solution of $\Delta F_{1}=\frac{\partial T_{1}}{\partial x} \cos \theta-\frac{\partial T_{1}}{\partial z} \sin \theta$ and $F_{2}$ solution of $\Delta F_{2}=$ $\frac{\partial T_{2}}{\partial x} \cos \theta-\frac{\partial T_{2}}{\partial z}(-\sin \theta)=\frac{\partial T_{1}}{\partial x} \cos \theta-\left(-\frac{\partial T_{1}}{\partial z}\right)(-\sin \theta)$, so that we see that $F_{1}$ and $F_{2}$ verify the same equation and $F_{2}=F_{1}$. Concerning the driving force $F_{v 1}$ for $\alpha=90^{\circ}-\theta$, it is given by

$$
F_{v 1}=\operatorname{Gr}_{\mathrm{v}}\left[\left(\frac{\partial^{2} F_{1}}{\partial x^{2}} \frac{\partial T_{1}}{\partial z}-\frac{\partial^{2} F_{1}}{\partial x \partial z} \frac{\partial T_{1}}{\partial x}\right) \cos \theta-\left(\frac{\partial^{2} F_{1}}{\partial x \partial z} \frac{\partial T_{1}}{\partial z}-\frac{\partial^{2} F_{1}}{\partial z^{2}} \frac{\partial T_{1}}{\partial x}\right) \sin \theta\right]
$$

whereas for $\alpha=90^{\circ}+\theta$, we have

$F_{v 2}=\operatorname{Gr}_{\mathrm{v}}\left[\left(\frac{\partial^{2} F_{2}}{\partial x^{2}} \frac{\partial T_{2}}{\partial z}-\frac{\partial^{2} F_{2}}{\partial x \partial z} \frac{\partial T_{2}}{\partial x}\right) \cos \theta-\left(\frac{\partial^{2} F_{2}}{\partial x \partial z} \frac{\partial T_{2}}{\partial z}-\frac{\partial^{2} F_{2}}{\partial z^{2}} \frac{\partial T_{2}}{\partial x}\right)(-\sin \theta)\right]$,

and then 


$$
\begin{aligned}
F_{v 2} & =\operatorname{Gr}_{\mathrm{v}}\left[\left(\frac{\partial^{2} F_{1}}{\partial x^{2}}\left(-\frac{\partial T_{1}}{\partial z}\right)-\left(-\frac{\partial^{2} F_{1}}{\partial x \partial z}\right) \frac{\partial T_{1}}{\partial x}\right) \cos \theta-\left(\left(-\frac{\partial^{2} F_{1}}{\partial x \partial z}\right)\left(-\frac{\partial T_{1}}{\partial z}\right)-\frac{\partial^{2} F_{2}}{\partial z^{2}} \frac{\partial T_{1}}{\partial x}\right)(-\sin \theta)\right] \\
& =-F_{v 1},
\end{aligned}
$$

i.e. the force $F_{v 2}$ for $\alpha=90^{\circ}+\theta$ is the opposite of $F_{v 1}$ for $\alpha=90^{\circ}-\theta$. Similar flows, but with opposite rotation direction, will then be generated in both cases in the limit of large values of $\mathrm{Gr}_{\mathrm{v}} / \mathrm{Gr}$. As a result, strong vibrations with directions that are either parallel, $\alpha=0^{\circ}$ (or $\alpha=$ $180^{\circ}$ ), or perpendicular, $\alpha=90^{\circ}$, to the thermal gradient, will lead to very weak flows and have to be avoided if the objective is to enhance the flow as in mixing applications and silicon purification processes. These vibration directions, however, are recommended for stabilizing the system.

Finally, according to (18), flow inversion can only occur for $\mathrm{Gr}_{\mathrm{v}} / \mathrm{Gr} \geq 2$. For smaller values of $\mathrm{Gr}_{\mathrm{v}} / \mathrm{Gr}$, vibrations will act on the buoyancy flow and modify its intensity, but will never invert the rotation direction of the main central roll, regardless of the vibration angle $\alpha$. The limit situation $\mathrm{Gr}_{\mathrm{v}} / \mathrm{Gr}=2$ corresponds to $\theta_{0}=90^{\circ}$ and then to $\alpha_{1}=\alpha_{2}=135^{\circ}$, i.e. the domain of flow inversion disappears at $\alpha=135^{\circ}$. The results obtained for $\mathrm{Gr}=2500$ and three values of $\mathrm{Gr}_{\mathrm{v}}\left(1250,3125\right.$ and 5000) are shown in figure 11 where we plot $\psi_{c}$ versus $\alpha$. The ratios $\mathrm{Gr}_{\mathrm{v}} / \mathrm{Gr}$ are then $0.5,0.8$ and 2. As expected, no flow inversion is observed in these cases. Note that for the limit theoretical value for flow inversion, $\mathrm{Gr}_{\mathrm{v}} / \mathrm{Gr}=2$, the results show that the curve of $\psi_{c}$ has its maximum value which is still negative, but very close to $\psi_{c}=0$ and reached for $\alpha$ close to $135^{\circ}$. This theoretical limit value is then a good approximation of the limit for flow inversion in our two-dimensional cavity.

Note finally that the theoretical model has been validated for a $A=4$ cavity and a melt with $\operatorname{Pr}=0.01$. We can expect that the assumptions on $F$ ( $F$ and its derivatives independent of $x$ in the central part of the cavity) will be well verified for large aspect ratio cavities and that those on $T$ (diffusive $T$ field) will benefit from small values of Pr.

\section{Conclusion}

In the present work, the convection induced in a silicon melt inside a rectangular crucible with vertical hot and cold walls and submitted to high frequency vibrations has been considered. Assuming the period of the vibrations to be much smaller than viscous and thermal diffusion times, the averaging method proposed by Gershuni and Lyubimov [2] has been used to derive the mean flow and temperature equations, which are then solved with a Chebyshev spectral collocation method. Theoretical and numerical studies for different values of the Grashof number $\mathrm{Gr}$ and vibrational Grashof number $\mathrm{Gr}_{\mathrm{v}}$ and all possible directions for the vibrations, from $0^{\circ}$ to $180^{\circ}$ with respect to the temperature gradient, have then been performed. Numerical results show that the global range of vibration angles from $\alpha=0^{\circ}$ to $\alpha=180^{\circ}$ can be divided in different regions for each set of parameters $\left(\mathrm{Gr}\right.$ and $\left.\mathrm{Gr}_{\mathrm{v}}\right)$. Vibration effect leads to a flow damping for small vibration angles and a flow enhancement for moderate angles, up to a maximal value reached for a critical angle $\alpha_{m}$ in the neighborhood of $45^{\circ}$. The decrease of the flow intensity beyond $\alpha_{m}$ can eventually lead for certain couples of parameters $\mathrm{Gr}$ and $\mathrm{Gr}_{\mathrm{v}}$ to flow inversion in a specified angle range $\left[\alpha_{1}, \alpha_{2}\right]$ inside $\left[90^{\circ}, 180^{\circ}\right]$.

A general theoretical expression has been derived for the limit vibration angles $\alpha_{1}$ and $\alpha_{2}$ defining the domain of flow inversion. We use the fact that flow inversion occurs when the global driving force is zero in the central part of the cavity, which also leads to specific 
properties of the different scalar fields involved. This theoretical expression gives the flow inversion angle range as a simple function of the ratio $\mathrm{Gr}_{v} / \mathrm{Gr}$. For any ratio $\mathrm{Gr}_{\mathrm{v}} / \mathrm{Gr}$, a very good agreement is found between the numerical results and the prediction of the theoretical model, with very close values of $\alpha_{1}$ and $\alpha_{2}$.

Two limiting cases have also been discussed. The case of very large values of the ratio $\mathrm{Gr}_{\mathrm{v}} / \mathrm{Gr}$ corresponds to the maximum $\alpha$ range, $\left[90^{\circ}, 180^{\circ}\right.$, for flow inversion and, in this limit, the reverse flow is as intense as the usual flow, with both flows connected by an up-down symmetry between states corresponding to vibration angles on both sides of $\alpha=90^{\circ}$. In contrast, for small values of $\mathrm{Gr}_{\mathrm{v}} / \mathrm{Gr}$, flow inversion cannot occur, up to the limit $\mathrm{Gr}_{\mathrm{v}} / \mathrm{Gr}=2$ given by the theoretical model and well reproduced by the numerical results.

\section{Acknowledgements}

This collaborative work was supported by the PHC Maghreb Partnership Program no. 36951NG. A grant of the PROFAS B+ program given to A.M. is also gratefully acknowledged.

\section{References}

[1] Savino R., Monti R. (1999) Improving diffusion controlled microgravity experiments by facility orientation, J. Aerospace Eng., 212, Part G, 415-426

[2] Gershuni G.Z., Lyubimov D.U. (1998) Thermal vibrational convection, Wiley, New York.

[3] Gresho P.M., Sani R.L. (1970) The effects of gravity modulation on the stability of a heated fluid layer, J. Fluid Mech., 40, 783-806.

[4] Gershuni G.Z., Zhukhovitskii E.M., Iurkov Y.S. (1970) On convective stability in the presence of periodically varying parameter, J. Appl. Math. Mech., 34, 470-480.

[5] Gershuni G.Z., Zhukhovitskii E.M., Iurkov Y.S. (1982) Vibrational thermal convection in a rectangular cavity, Fluid Dyn., 17(4), 565-569.

[6] Biringen S., Peltier L.J. (1990) Numerical simulation of 3-D Bénard convection with gravitational modulation, Phys. Fluids A, 2, 754-764.

[7] Uspenskii V., Favier J.J. (1994) High frequency vibration and natural convection in Bridgman-scheme crystal growth, Int. J. Heat Mass Transfer, 37(4), 691-698.

[8] Savino R., Monti R., Piccirillo M. (1998) Thermovibrational convection in a fluid cell, Comput. Fluids, 27(8), 923-939.

[9] Lan.C.W (2000) Effects of axial vibration on vertical zone-melting processing, Int. J. Heat Mass Transfer, 43, 1987-1997.

[10] Straube A.V., Lyubimov D.V., Shklyaev S.V. (2006) Averaged dynamics of two-phase media in a vibration field, Phys. Fluids, 18, 053303.

[11] Chang Q., Iwan J., Alexander D. (2007) Thermal vibrational convection in a two-phase stratified liquid, C. R. Mécanique, 335, 304-314.

[12] O’Hern T.J., Shelden B., Torczynski J.R., Romero L.A. (2012) Bubble oscillations and motion under vibration, Phys. Fluids, 24, 091108. 
[13] Shevtsova V., Ryzhkov I., Melnikov D. E., Gaponenko Y.A., Mialdun A. (2010) Experimental and theoretical study of vibration-induced thermal convection in low gravity, J. Fluid Mech., 648, 53-82.

[14] Ferrera C., Herrada M.A., Montanero J.M., Torregrosa M., and Shevtsova V. (2014) Dynamical response of liquid bridges to a step change in the mass force magnitude, Phys. Fluids, 26, 012108.

[15] Gaponenko M., Mialdun A., Shevtsova V. (2018) Pattern selection in miscible liquids under periodic excitation in microgravity: Effect of interface width, Phys. Fluids, 30, 062103 .

[16] Lappa M. (2013) On the variety of particle accumulation structures under the effect of g-jitters, J. Fluid Mech., 726, 160-195.

[17] Lappa M. (2016) Control of convection patterning and intensity in shallow cavities by harmonic vibrations, Microgravity Sci. Technol., 28, 29-39.

[18] Lappa M. (2016) Patterning behavior of gravitationally modulated supercritical Marangoni flow in liquid layers, Phys. Rev. E, 93, 053107.

[19] Savino R., Lappa M. (2003) Assessment of the thermovibrational theory: application to g-jitter on the space station, J. Spacecraft Rock., 40(2), 201-210.

[20] Peyret R. (2002) Spectral Methods for Incompressible Viscous Flow, App. Math. Sciences, 148, Springer-Verlag, New York.

[21] Trefethen N. L. (2000) Spectral methods in Matlab, SIAM, Philadelphia.

[22] Weideman J.A.C., Reddy S.C. (2000) A Matlab differentiation matrix suite, ACM Transactions on Mathematical Software, 26(4), 465-519.

[23] Phillips T. N. (1984) Natural convection in an enclosed cavity, J. Comp. Phys., 54(3), 365-381. 


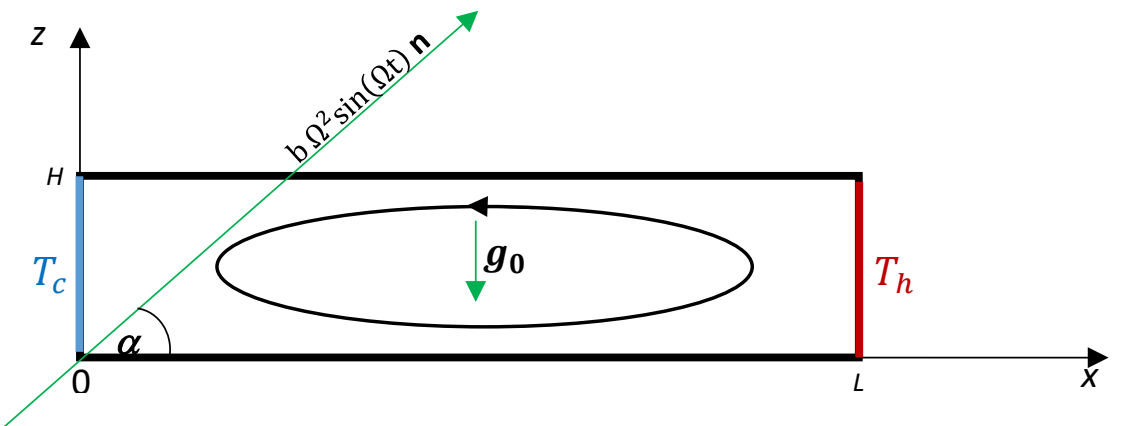

Figure 1: Schematic of the crucible subject to thermal vibration. The left and right sidewalls are respectively cooled and heated. 


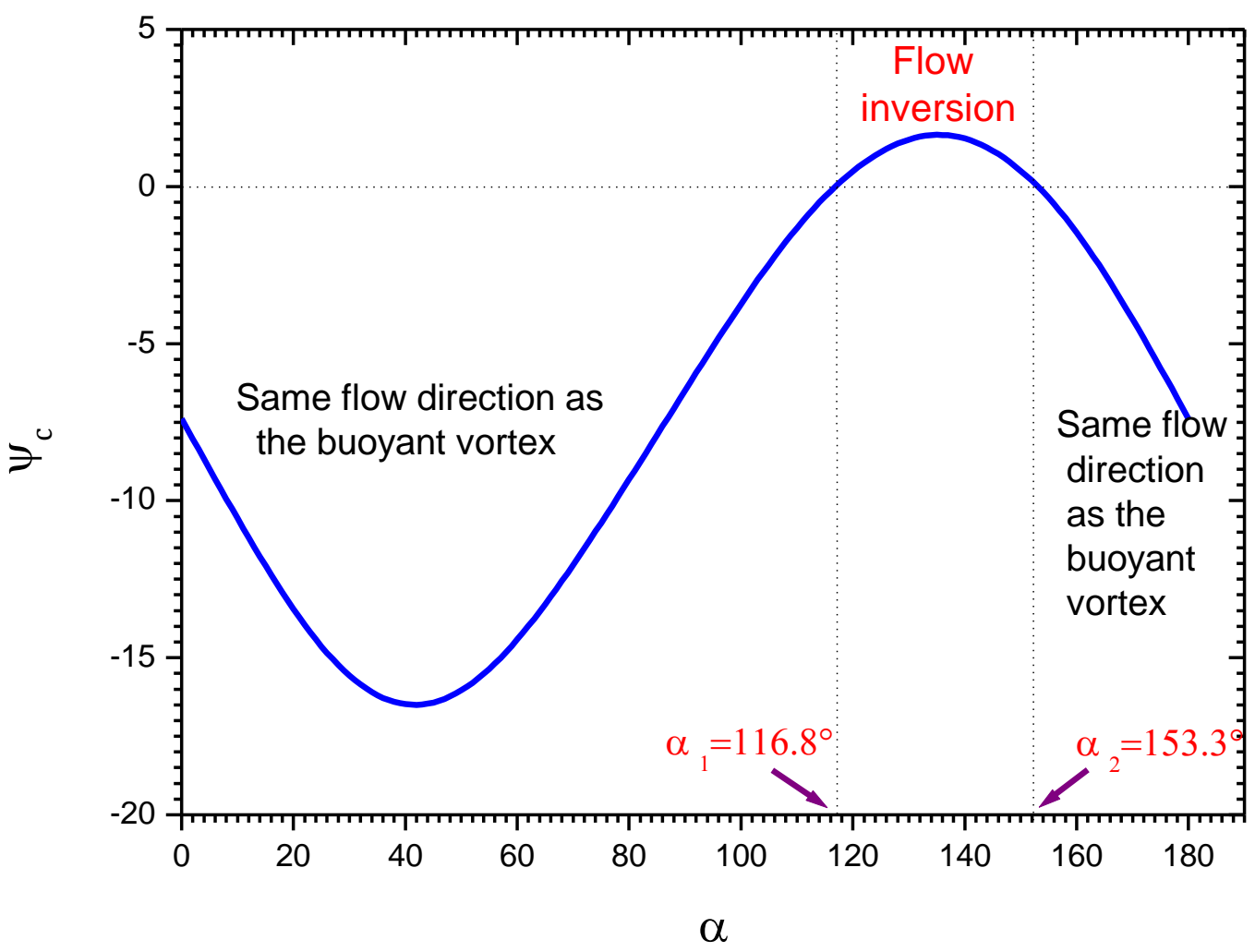

(a)

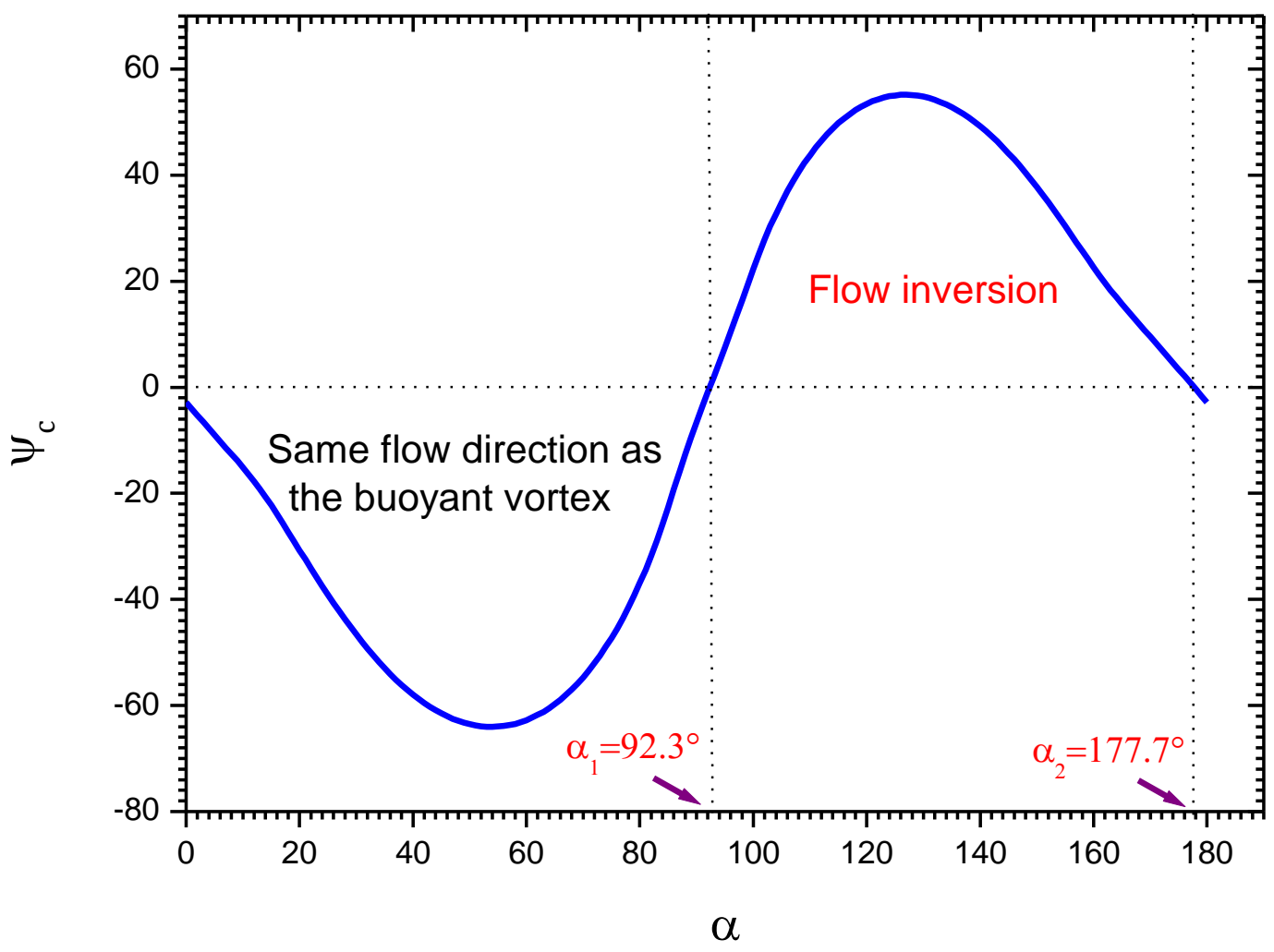

(b)

Figure 2: Stream function at the center of a $A=4$ side-heated cavity submitted to vibrations as a function of the vibration angle $\alpha$ for $\operatorname{Pr}=0.01$ and $\mathrm{Gr}=2500$ : (a) $\mathrm{Gr}_{\mathrm{v}}=6.2510^{3}$; (b) $\mathrm{Gr}_{\mathrm{v}}=6.2510^{4}$. 


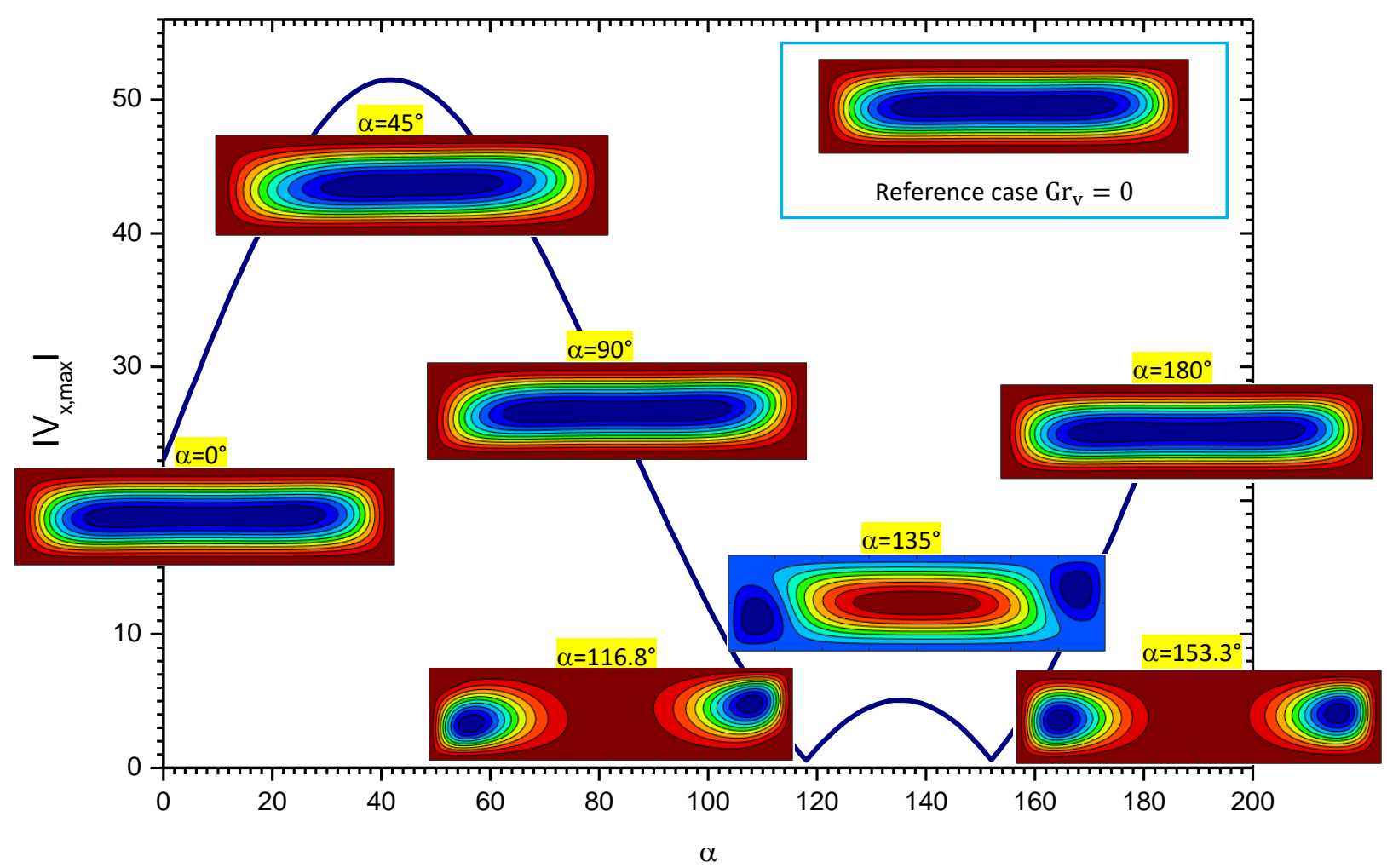

Figure 3: Maximal absolute horizontal velocity in a $A=4$ side-heated cavity submitted to vibrations as a function of the vibration angle $\alpha$ for $\operatorname{Pr}=0.01, \mathrm{Gr}=2500$ and $\mathrm{Gr}_{\mathrm{v}}=6.2510^{3}$. Insets give the streamfunction $\psi$ in the cavity for selected values of $\alpha$. 


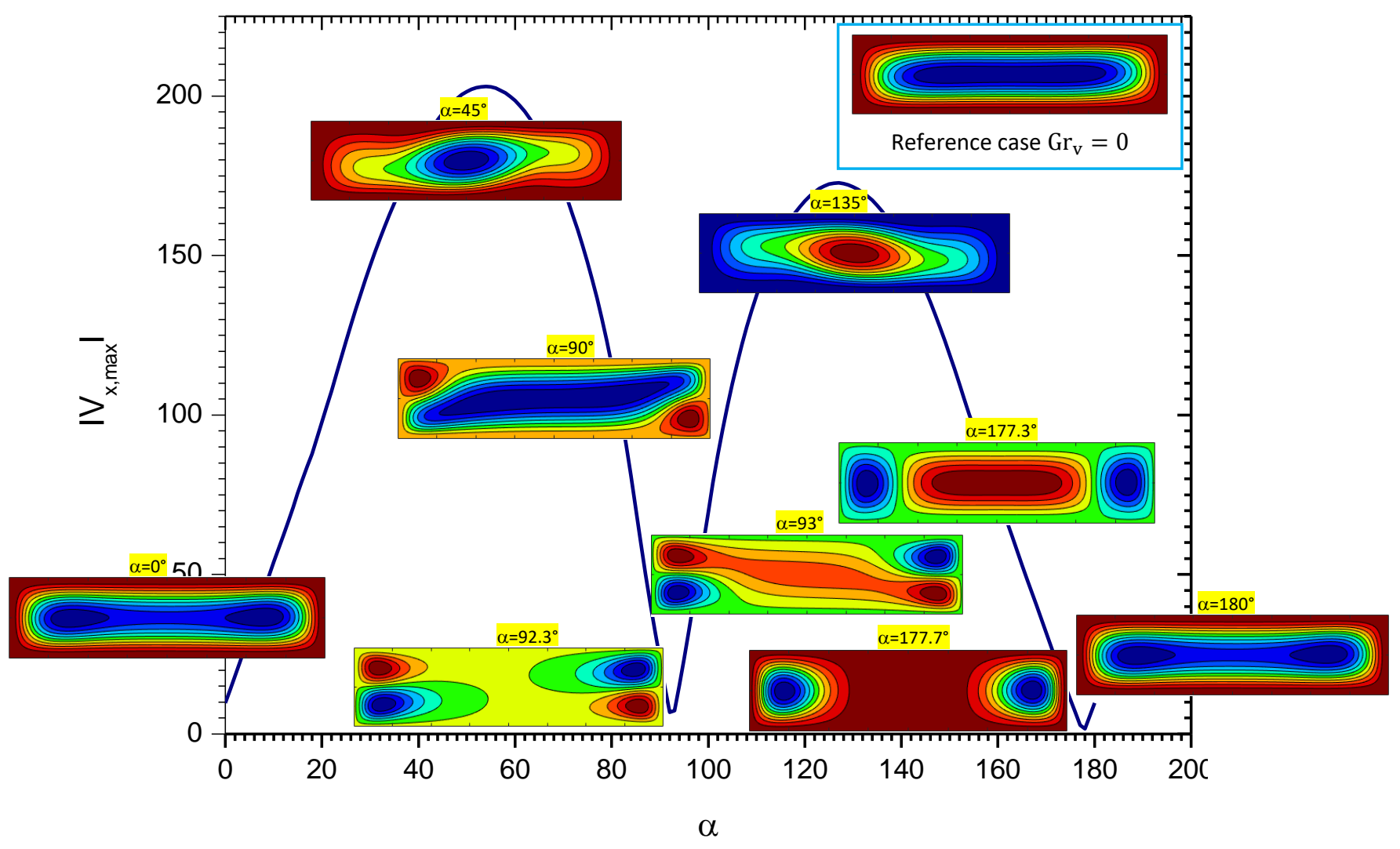

Figure 4: Maximal absolute horizontal velocity in a $A=4$ side-heated cavity submitted to vibrations as a function of the vibration angle $\alpha$ for $\operatorname{Pr}=0.01, \mathrm{Gr}=2500$ and $\mathrm{Gr}_{\mathrm{v}}=6.2510^{4}$. Insets give the streamfunction $\psi$ in the cavity for selected values of $\alpha$. 

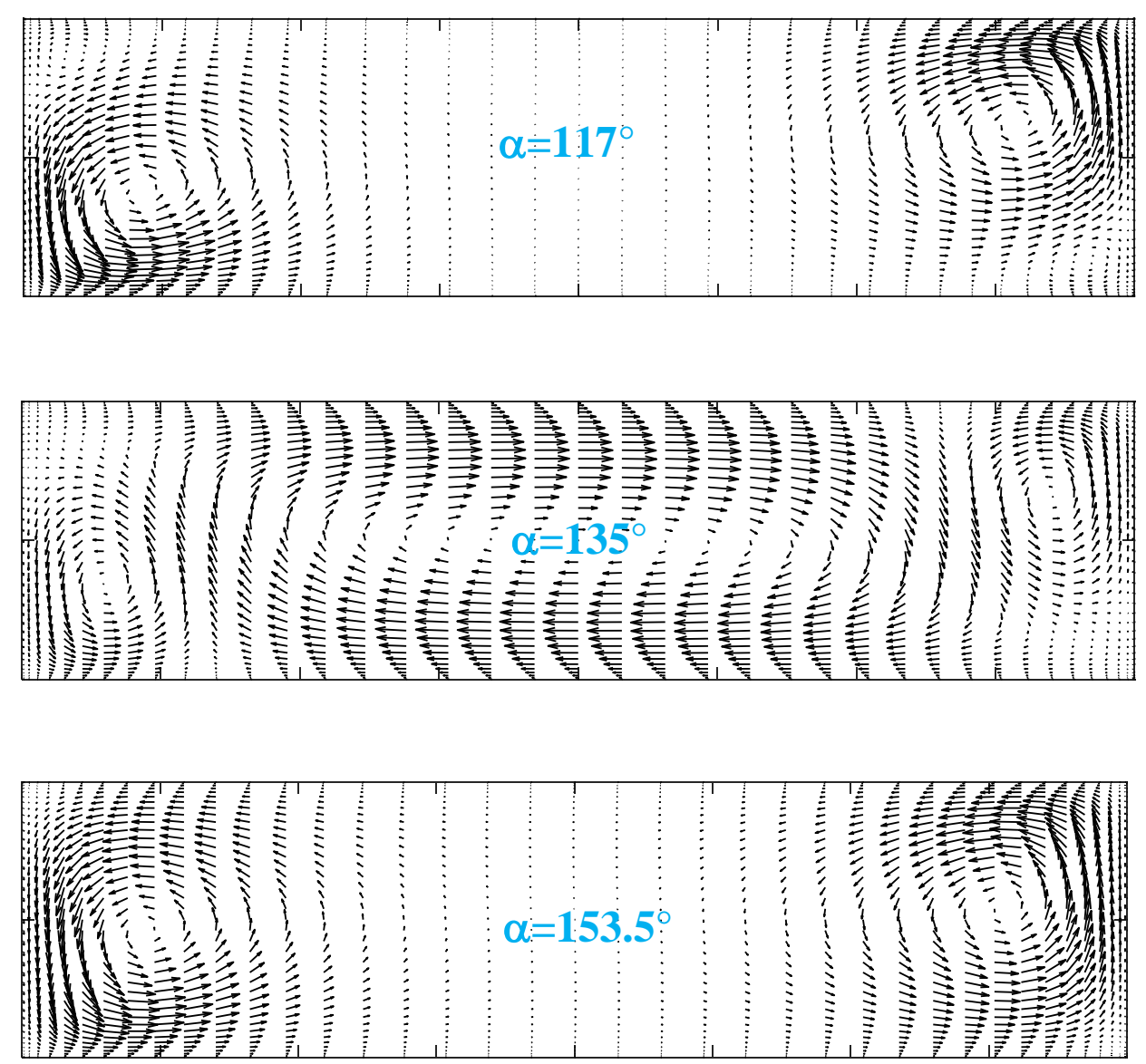

Figure 5: Velocity vectors fields in a $A=4$ side-heated cavity submitted to vibrations for selected vibration angles, $\alpha=117^{\circ}, 135^{\circ}$ and $153.5^{\circ}\left(\mathrm{Gr}=2500, \mathrm{Gr}_{\mathrm{v}}=6.2510^{3}, \mathrm{Pr}=\right.$ 0.01). 

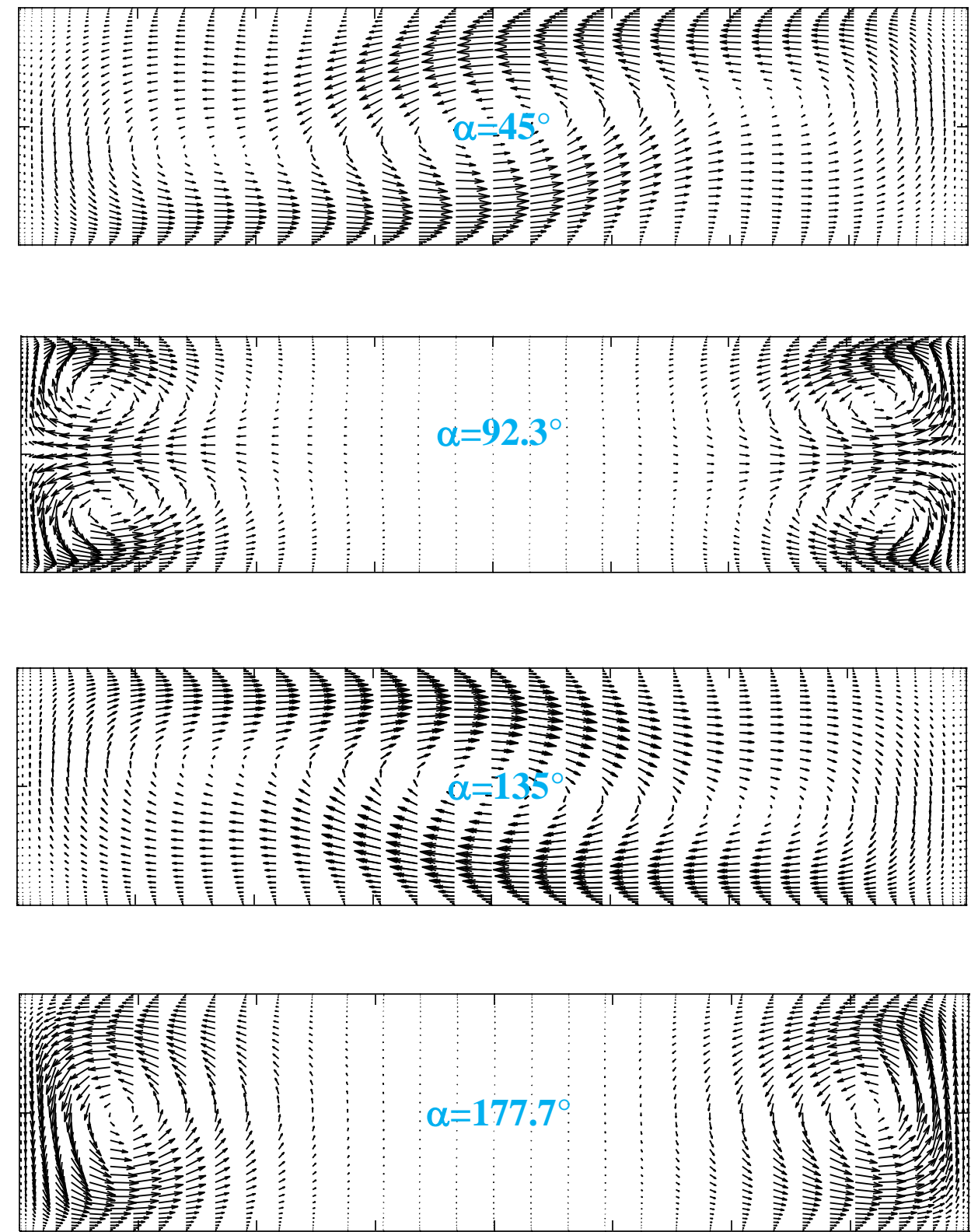

Figure 6: Velocity vectors fields in a $A=4$ side-heated cavity submitted to vibrations for selected vibration angles, $\alpha=45^{\circ}, 92.3^{\circ}, 135^{\circ}$ and $177.7^{\circ}\left(\mathrm{Gr}=2500, \mathrm{Gr}_{\mathrm{v}}=6.2510^{4}\right.$, $\operatorname{Pr}=0.01)$. 


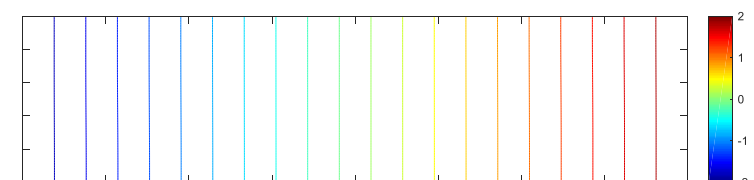

(a)

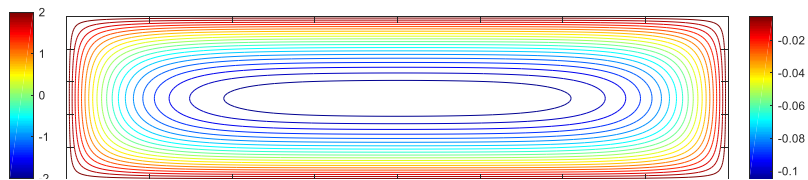

(b)

Figure 7: Isotherms (a) and iso- $F$ lines (b) in a $A=4$ side-heated cavity submitted to vibrations for the case $\operatorname{Pr}=0.01, \mathrm{Gr}=2500$ and $\mathrm{Gr}_{\mathrm{v}}=6250$ with a vibration angle $\alpha=117^{\circ}$, i.e. close to the limit of flow inversion $\alpha_{1}$. 


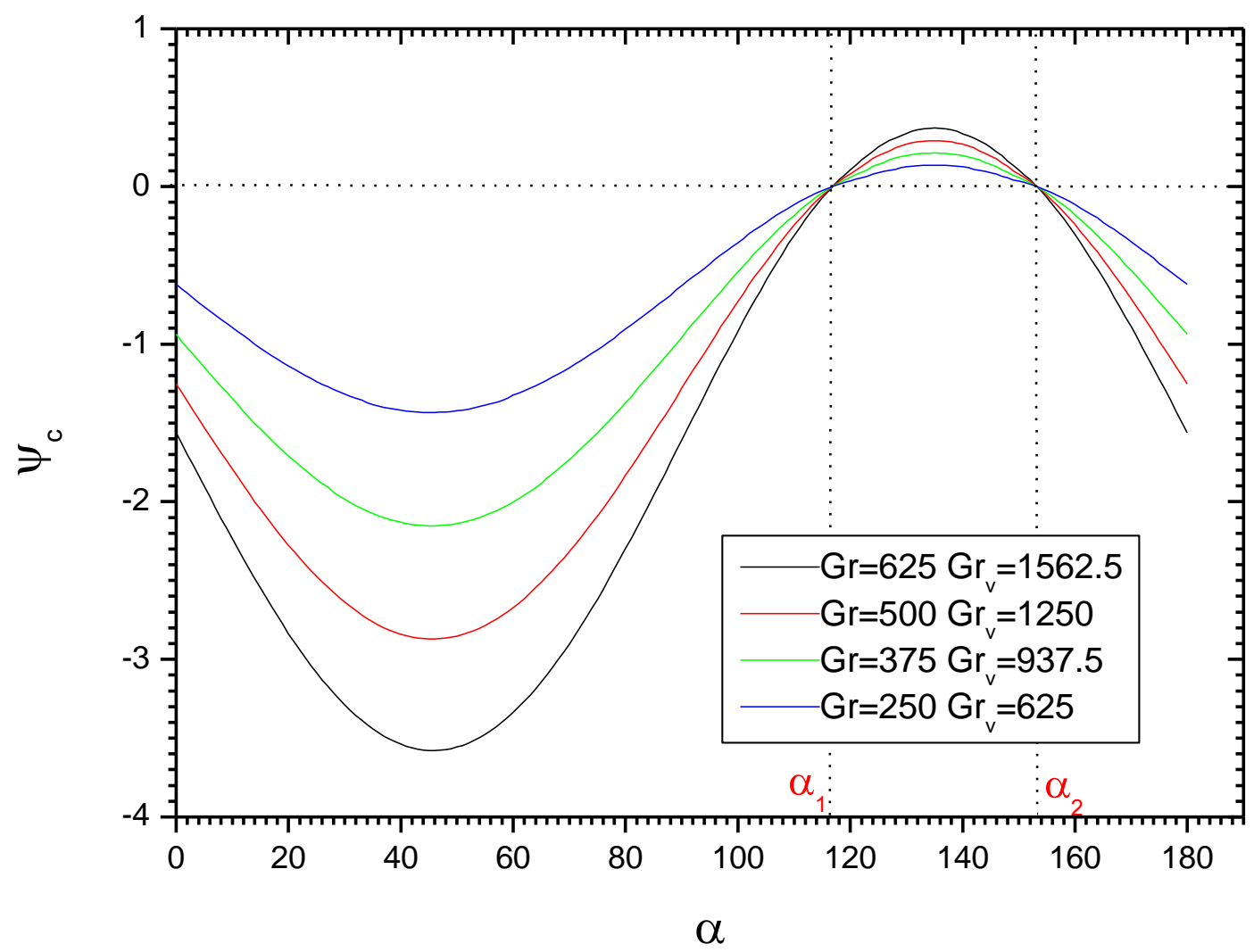

Figure 8: Stream function at the center of a $A=4$ side-heated cavity submitted to vibrations as a function of the vibration angle $\alpha$ for $\operatorname{Pr}=0.01$ and different values of $\mathrm{Gr}$ and $\mathrm{Gr}_{\mathrm{v}}$ corresponding to $\mathrm{Gr}_{\mathrm{v}} / \mathrm{Gr}=2.5$. Flow inversion occurs for the same values of $\alpha_{1}$ and $\alpha_{2}$. 


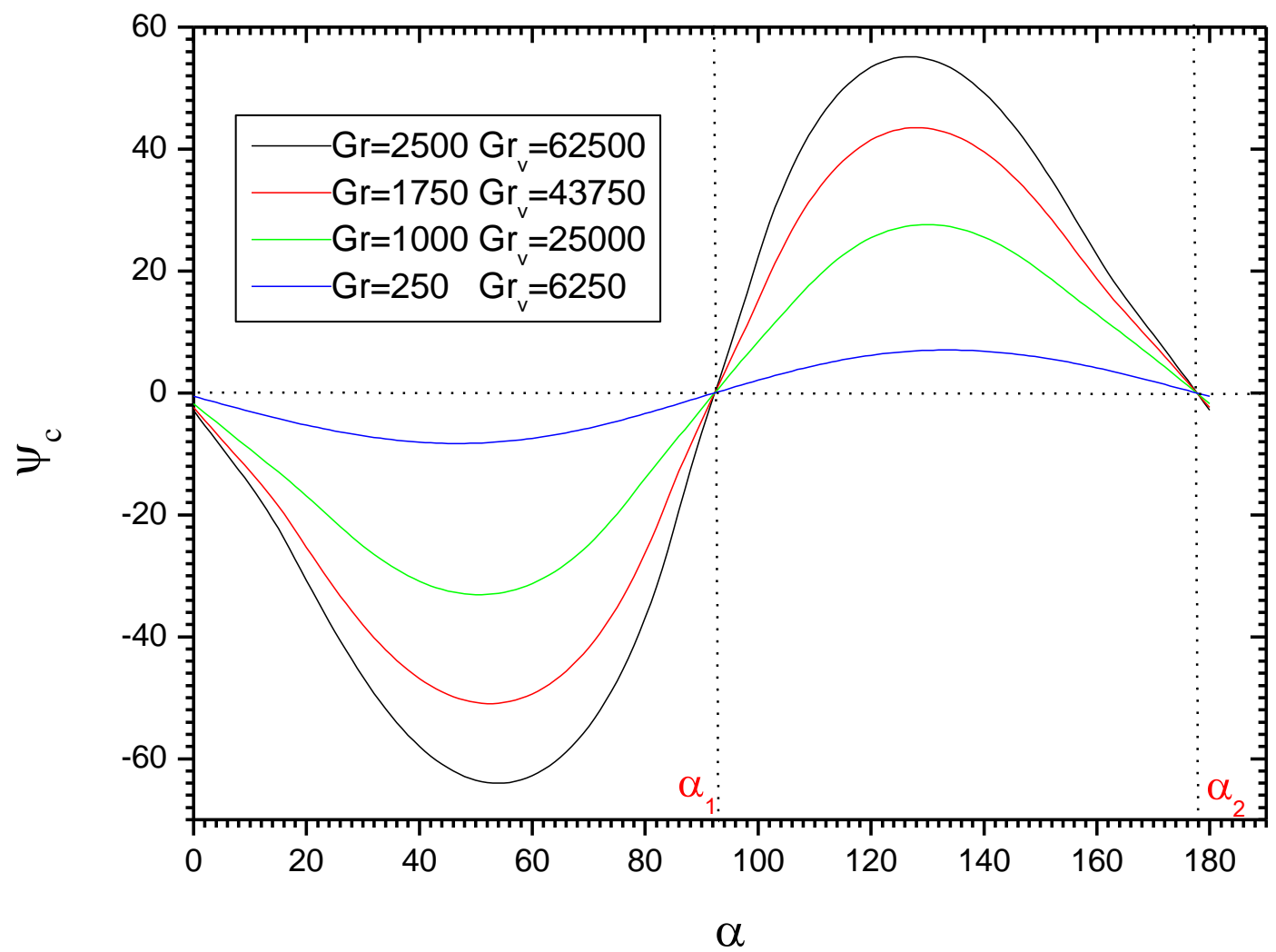

Figure 9: Stream function at the center of a $A=4$ side-heated cavity submitted to vibrations as a function of the vibration angle $\alpha$ for $\operatorname{Pr}=0.01$ and different values of $\mathrm{Gr}$ and $\mathrm{Gr}_{\mathrm{v}}$ corresponding to $\mathrm{Gr}_{\mathrm{v}} / \mathrm{Gr}=25$. Flow inversion occurs for the same values of $\alpha_{1}$ and $\alpha_{2}$. 


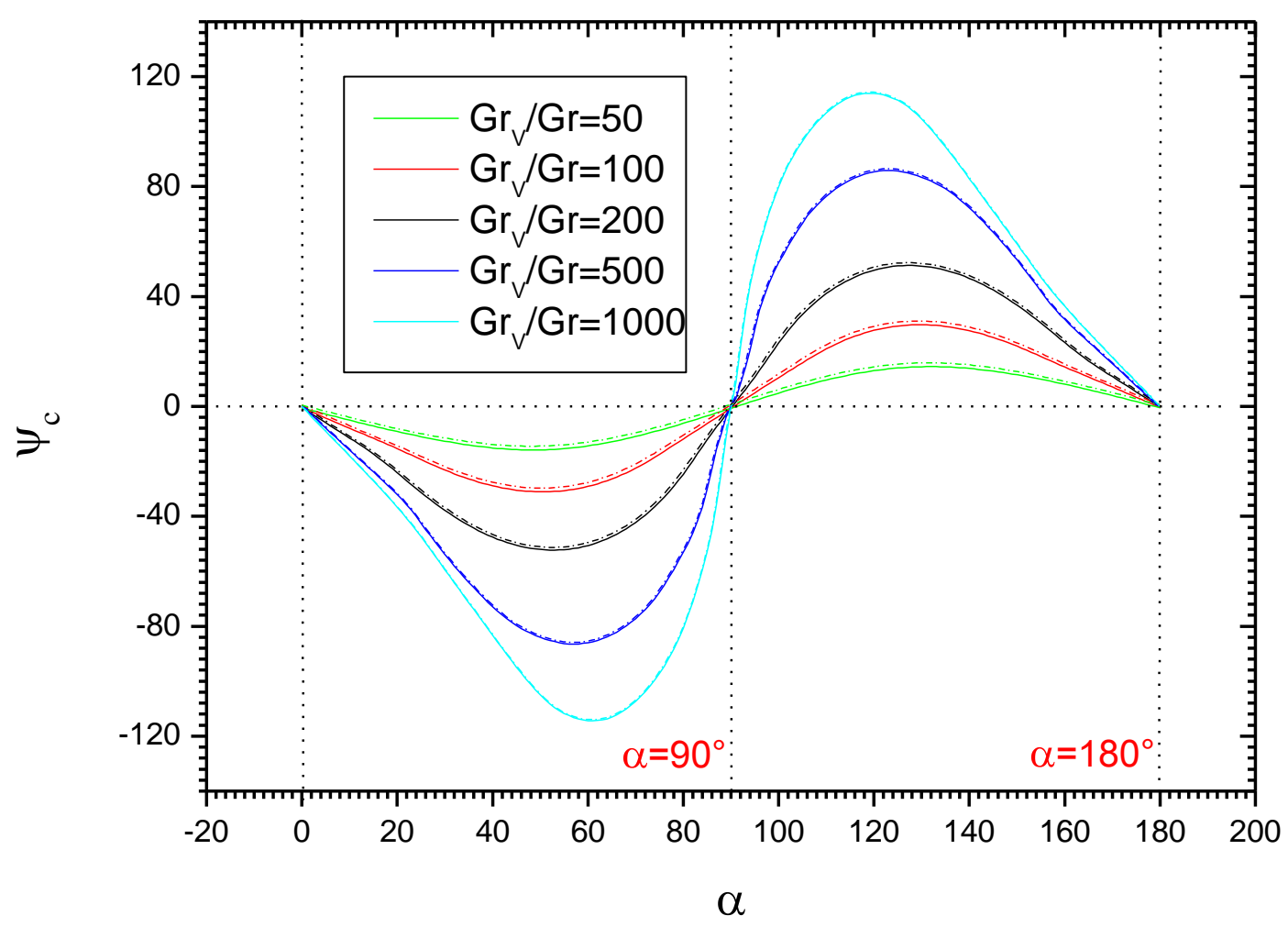

Figure 10: Stream function at the center of a $A=4$ side-heated cavity submitted to vibrations as a function of the vibration angle $\alpha$ for $\mathrm{Pr}=0.01, \mathrm{Gr}=250$ and $\mathrm{Gr}_{\mathrm{v}}=12500,25000$, 50000, 125000 and 250000 corresponding respectively to $\mathrm{Gr}_{\mathrm{v}} / \mathrm{Gr}=50,100,200,500$ and 1000. $\psi_{c}(\alpha)$ is given as solid lines. In order to compare the intensity of the counterclockwise and clockwise flows, $-\psi_{c}\left(180^{\circ}-\alpha\right)$ is also plotted as dashed lines. For these strong values of $\mathrm{Gr}_{\mathrm{v}} / \mathrm{Gr}$, flow inversion occurs for values of $\alpha_{1}$ and $\alpha_{2}$ very close to $90^{\circ}$ and $180^{\circ}$, respectively, and the intensities of the counterclockwise and clockwise flows are very similar. 


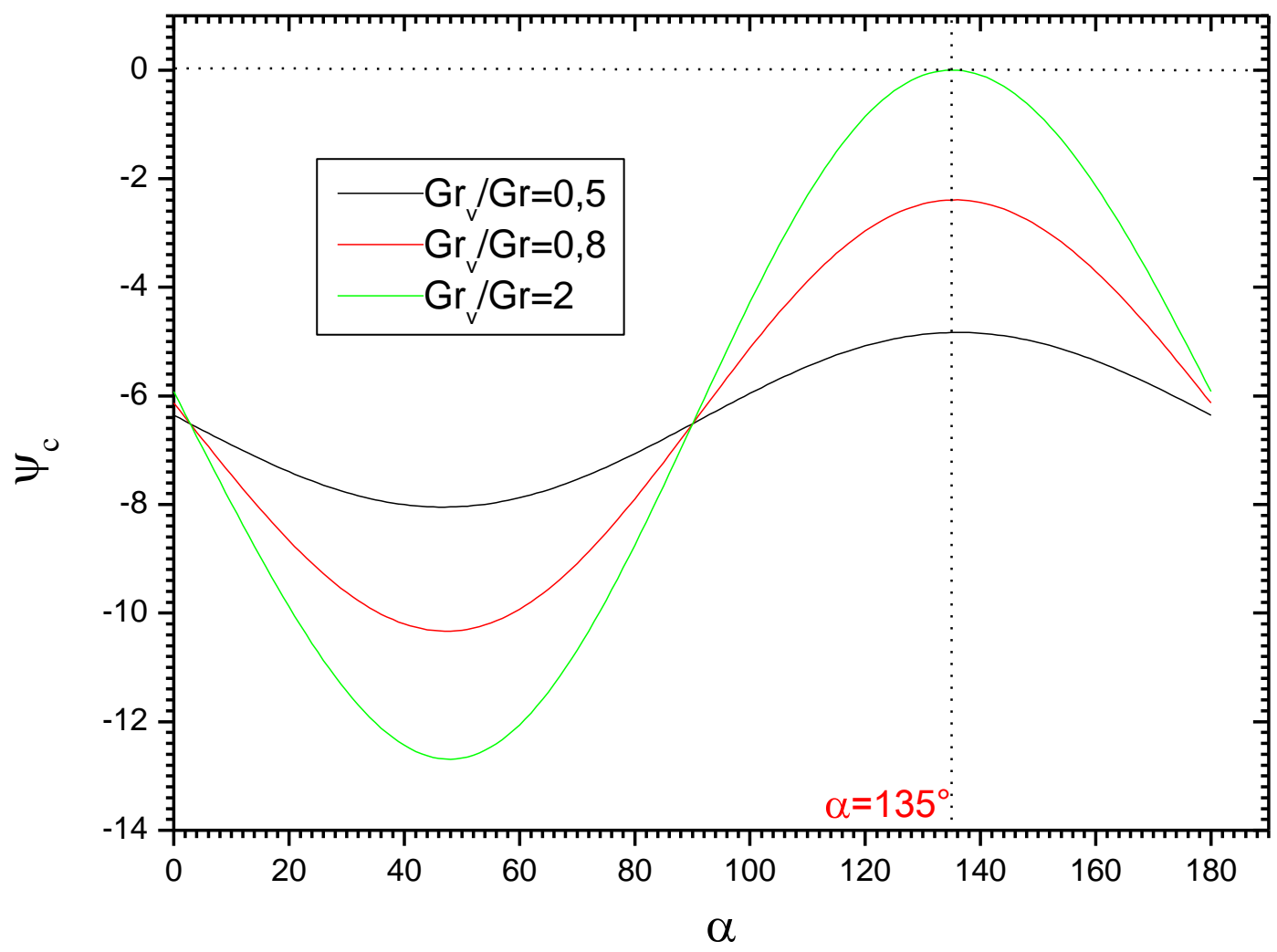

Figure 11: Stream function at the center of a $A=4$ side-heated cavity submitted to vibrations as a function of the vibration angle $\alpha$ for $\operatorname{Pr}=0.01, \mathrm{Gr}=2500$ and $\mathrm{Gr}_{\mathrm{v}}=1250,3125$ and 5000. The ratios $\mathrm{Gr}_{\mathrm{v}} / \mathrm{Gr}$ are then 0.5, 0.8 and 2. No flow inversion occurs in these cases, but the case at $\mathrm{Gr}_{\mathrm{v}} / \mathrm{Gr}=2$ is at the limit of flow inversion with a maximum value of $\psi_{c}$ close to zero at $\alpha=135^{\circ}$, as predicted by our model. 